\title{
Comparative analysis of the rectal and caecal microbial community composition and function in adult Erhualian and Sushan pigs
}

\author{
B. Li ${ }^{1}$, S. Ren ${ }^{1}$, W. Zhao, X. Wang and F. Tu \\ Jiangsu Academy of Agricultural Sciences, Institute of Animal Science, Zhongling Street 50, Xuanwu, Nanjing, Jiangsu, China
}

KEY WORDS: fat deposition, intestinal microorganisms, nutrient absorption, pig breeds

Received: 13 April 2021

Revised: 2 June 2021

Accepted: 10 June 2021

${ }^{1}$ Corresponding author:

e-mail: 1026563909@qq.com;

shouwenren@163.com

\begin{abstract}
Intestinal microbiota plays an important role in nutrition, metabolism and immunity in all mammals. It is comprised of diverse populations of bacteria and other microorganisms whose abundances are impacted by both environmental and host genetic factors. However, the understandings of the intestinal microbiota in different pig breeds remain largely undefined. To examine the differences in intestinal microflora between two pig breeds with different genetic backgrounds under the same environment, 16S rRNA gene amplification and sequencing were performed to investigate the structural composition and potential functions of microbial communities in rectum and caecum of Erhualian and Sushan pigs. The results revealed that the diversity of intestinal microflora in two pig breeds was similar, but the abundance of specific intestinal microflora was different. At the phylum level, the dominant bacteria in caecum and rectum of Erhualian and Sushan pigs were Firmicutes, Acidobacteria and Bacteroides, but their expression abundance was different. Firmicutes and Bacteroidetes in Erhualian pigs were higher than those in Sushan pigs. At the genus level, Lactobacillus was the most abundant in caecum of Sushan pigs (6.83\%) and rectum of Erhualian pigs (9.61\%), while Ruminococcaceae UCG-005 were dominant in caecum of Erhualian pigs (10.89\%) and Streptococcus in rectum of Sushan pigs $(24.89 \%)$. This study further confirmed the existence of specific microbial community diversity and abundance in different pig breeds. The microbial community diversity and abundance in Erhualian and Sushan pigs were closely related to pig fat deposition and nutrient absorption.
\end{abstract}

\section{Introduction}

The stable microbial diversity of the pig intestine can promote the absorption of nutrients, prevent the occurrence of diseases, and promote the growth and development of pigs (Bergen, 2015). There is a close correlation between the composition of intestinal microflora and intestinal development, immune characteristics, glucose and lipid metabolism and meat quality (Choy et al., 2014). The intestinal microbiota plays an important role in nutrient digestion. For example, the abundances of Anaerofustis and Robinsoniella in sow faecal samples were positively correlated with the apparent crude fibre digestibility (Niu et al., 2019). Clostridium is associated with dietary fibre metabolism, and Turicibacter is correlated with butyric acid (Woting et al., 2014). Pig breed is also an important factor affecting intestinal microbial diversity. Yang et al. (2014) showed that there were differences in intestinal microflora among different breeds of pigs. There is a high similarity among the intestinal microbes 
of Landrace, Yorkshire and Duroc pigs. However, Bama mini, Erhualian and Xiaomeishan pigs from Chinese local breeds have high similarity when it comes to microorganisms presence and type. Diao et al. (2016) showed that intestinal microbial abundances in Rongchang, Tibetan and Landrace pigs were different. Tibetan and Rongchang pigs had a higher proportion of Firmicutes and Spirochaetes and a lower proportion of Bacteroidetes than Landrace pigs, and the proportion of Spirochaetes in Tibetan pigs was significantly higher than that in Rongchang pigs. Compared with Tibetan pigs, Landrace and Rongchang pigs contained a higher proportion of Tenericutes and a lower proportion of Fibrobacteres and Elusimicrobia. The above results indicated that the composition of intestinal microorganisms in pigs was probably related to the host's metabolic type, feeding characteristics and immune function.

Erhualian pig is a well-known local pig breed in China, with strong lactation, good motherhood, a high feeding rate, early sexual maturity, especially high resistance to rough feeding and a docile temperament. Sushan pig, as a new hybrid breed of Erhualian and Yorkshire pigs, has the advantages of delicious meat and a certain level of resistance to rough feeding. In pig production, the tolerance to rough feeding and crude fibre of Sushan pigs was found to be lower than that of Erhualian pigs, and the demand of Sushan pigs for nutrients in feed is higher than that of Erhualian pigs. In order to study the difference of tolerance to rough feeding and crude fibre between Erhualian and Sushan pigs, characterization and comparative analysis were performed to investigate the structural composition and potential functions of microbial communities in these two breeds. This study provides a powerful theoretical basis on the potential roles of intestinal microbial communities in resistance to rough feeding, nutrition metabolism and crude fibre digestion for safe and healthy pork production.

\section{Material and methods}

This experiment was reviewed and approved by the Institutional Animal Ethics Committee from the Research Integrity and Ethics Administration of Jiangsu Academy of Agricultural Sciences, China.

\section{Animals and sampling}

Erhualian and Sushan pigs (five barrows each) were fed at the Sushan Pig Breeding Farm (Nanjing, China) under the same conditions and provided with standard diets in accordance with the feeding standard of swine (NY/T 65-2004) issued by the Ministry of Agriculture of the People's Republic of China (Table 1). At the rapid growth stage $\left(175^{\text {th }}\right.$ day of age), the adult Erhualian $(75 \mathrm{~kg})$ and Sushan $(90 \mathrm{~kg})$ pigs were slaughtered according to standard procedures. The pigs were dissected for collecting the intestinal contents of the rectum and caecum. The samples were kept at $-80^{\circ} \mathrm{C}$ for $16 \mathrm{~S}$ rRNA gene analysis.

Table 1. Composition and nutrient levels of basal diets (air-dry basis), \%

\begin{tabular}{|c|c|}
\hline Indices & Conten \\
\hline \multicolumn{2}{|l|}{ Ingredients } \\
\hline maize & 61.00 \\
\hline soyabean meal & 9.00 \\
\hline wheat bran & 27.50 \\
\hline $\mathrm{CaHPO}_{4}$ & 0.50 \\
\hline limestone & 0.50 \\
\hline $\mathrm{NaCl}$ & 0.50 \\
\hline premix $^{1}$ & 1.00 \\
\hline \multicolumn{2}{|l|}{ Nutrient levels ${ }^{2}$} \\
\hline DM & 85.43 \\
\hline ash & 6.24 \\
\hline $\mathrm{CP}$ & 15.41 \\
\hline EE & 4.31 \\
\hline CF & 3.02 \\
\hline $\mathrm{DE}, \mathrm{MJ} / \mathrm{kg}$ & 18.32 \\
\hline $\mathrm{Ca}$ & 0.54 \\
\hline$P$ & 0.47 \\
\hline
\end{tabular}

DM - dry matter, CP - crude protein, EE - ether exract, CF - crude fibre, DE - digestible energy; ${ }^{1}$ the premix provided the following per $\mathrm{kg}$ of diets: mg: Fe 100, Zn 100, Mn 30, Cu 10, Se 0.3, I 0.5, vit. K 3.0, vit. $B_{1} 2.0$, vit. $B_{2} 6.0$, vit. $B_{6} 3.0$, nicotinic acid 30 , pantothenic acid 30 , folic acid 1.0, biotin 0.2, choline 300; IU: vit. A 8000 , vit. D 31000 , vit. E 20; $\mu$ g: vit. $B_{12} 30 ;{ }^{2}$ DE was calculated, while the other values were calculated

\section{DNA extraction and PCR amplification}

Microbial DNA was extracted from rectal and caecal samples using an E.Z.N.A. ${ }^{\circledR}$ soil DNA Kit (Omega Bio-tek, Norcross, GA, USA) according to the manufacturer's protocols. The final DNA concentration and purification were determined by a NanoDrop 2000 UV-Vis spectrophotometer (Thermo Scientific, Waltham, MA, USA), and the DNA quality was checked by $1 \%$ agarose gel electrophoresis. The V3/V4 hypervariable regions of the bacterial 16S rRNA gene were amplified using primers 341F (5'-CCTAYGGGRBGCASCAG-3') and 806R (5'-GGACTACNNGGGTATCTAAT-3') based on a thermocycler PCR system (GeneAmp PCR System 9700, Applied Biosystem, Foster City, CA, USA). The PCRs were conducted with the following program: initial denaturation at $94{ }^{\circ} \mathrm{C}$ for $4 \mathrm{~min}$; $94{ }^{\circ} \mathrm{C}$ denaturation for $30 \mathrm{~s}, 50{ }^{\circ} \mathrm{C}$ annealing for $45 \mathrm{~s}$ and $72{ }^{\circ} \mathrm{C}$ extension for $30 \mathrm{~s}$, repeated for 25 cycles; 
and final extension at $72{ }^{\circ} \mathrm{C}$ for $5 \mathrm{~min}$. PCRs were performed in triplicate with $20 \mu \mathrm{l}$ of a mixture containing $4 \mu \mathrm{l}$ of $5 \times$ FastPfu Buffer (Transgen Biotech, Beijing, China), $2 \mu \mathrm{l}$ of $2.5 \mathrm{mM}$ dNTPs, $0.8 \mu \mathrm{l}$ of each primer $(5 \mu \mathrm{m}), 0.4 \mu \mathrm{l}$ of FastPfu Polymerase, and $10 \mathrm{ng}$ of template DNA. The final PCR products were extracted from a $\%$ agarose gel, further purified with an AxyPrep DNA Gel Extraction Kit (Axygen Biosciences, Union City, CA, USA), and quantified using a QuantiFluor TM-ST (Promega, Madison, WI, USA) according to the manufacturer's protocol.

\section{Illumina MiSeq sequencing}

The purified amplicons were pooled in equimolar amounts and paired-end sequenced $(2 \times 300 \mathrm{bp})$ on an Illumina MiSeq platform (Illumina, San Diego, CA, USA) according to the standard protocols of the Majorbio Bio-Pharm Technology Co., Ltd. (Shanghai, China). The raw reads were deposited into the NCBI Sequence Read Archive (SRA) database (Accession Number: SRP279885).

\section{Data processing}

Raw FastQ files were demultiplexed, qualityfiltered using a fastp (version 0.20.0, https://github. com/OpenGene/fastp; Chen et al., 2018), and merged using a FLASH (version 1.2.7, http://ccb.jhu.edu/ software/FLASH; Magoč and Salzberg, 2011) with the following criteria: (i) the reads were truncated at any site receiving an average quality score $<20$ over a $50 \mathrm{bp}$ sliding window; (ii) primers were accurately matched, allowing 2 nucleotides to be mismatched, and reads containing ambiguous bases were removed; and (iii) sequences whose overlap was longer than $10 \mathrm{bp}$ were merged according to their overlap sequence.

Operational taxonomic units (OTUs) were clustered with a $97 \%$ similarity cut-off using a UPARSE (version 7.1, http://drive5.com/uparse; Edgar, 2013), and chimeric sequences were identified and removed using a UCHIME (version 4.2.40, http://www.drive5. com/uchime). The taxonomy of each 16S rRNA gene sequence was analysed with an RDP Classifier algorithm (http://rdp.cme.msu.edu) against the SILVA (SSU123) 16S rRNA database at a confidence threshold of $70 \%$.

\section{Data analysis}

Community diversity at the inter- and intragroup levels was assessed using a combination of bias Sobs, Shannon diversity indices, Simpson's diversity index, the abundance-based coverage estimator (ACE), the Chaol richness estimator and the coverage percentage. Based on the OTU expression profile, the alpha diversity of different samples at the OTU level was calculated. All of the aforementioned analyses were conducted using a MOTHUR (Kemp and Aller, 2004; Schloss et al., 2009). The principal component analysis (PCA) was performed based on the expression profile of OTUs at the taxonomic level using the R package (R Core Team, 2020). To identify differentially abundant taxa in multiple segments within different pig breeds, the linear discriminant analysis (LDA) effect size (LEFse) method was applied (Segata et al., 2011). To identify differentially abundant microbial taxa in the same segment among samples of different pig breeds a Metastats was used (White et al., 2009). Phylogenetic investigation of communities by reconstruction of unobserved states (PICRUS) (Langille et al., 2013) was applied to predict the functional enrichment of the microbial communities against the Kyoto Encyclopedia of Genes and Genomes (KEGG) database (Du et al., 2014). Correlation coefficients of the pathway enrichment for the samples of different pig breeds were calculated by the Spearman method. The $\mathrm{R}$ package 3.3.1, edgeR (Robinson et al., 2010), was used to determine differentiating molecular functions and pathways with a threshold of $\log 2$ fold change $>2$ and FDR $<0.01$. Volcano plots and heatmaps were generated for the differentiating pathways.

\section{Results}

\section{Species annotation and assessment}

OTU analysis. In total 1346719 tags were obtained from all samples, covering 560597784 base pairs (Table 2). The average tag count per sample was 67 336, and 1397 OTUs at 97\% identity were obtained, with the number of OTUs ranging from 523 to 1395 per sample. Coverage was determined to be greater than $95 \%$ in each sample. The sparse curve showed an obvious asymptote, which indicated that the sampling of the microbial community was close to complete and the sequencing depth was sufficient for diversity evaluation.

Alpha diversity analysis. The results of Sobs and Chao 1 indices showed that the relative abundances of bacteria in rectum were higher than those in caecum, and the relative abundances of bacteria in rectum and caecum of Sushan pigs were higher than those of Erhualian pigs. The results of Shannon and Simpson indices showed that the bacterial community diversity of rectum was higher than that of caecum in Erhualian pigs, while an opposite result was found in Sushan pigs (Table 3). 
Table 2. Tag number and length of the segmented samples in rectum and caecum of Erhualian and Sushan pigs

\begin{tabular}{|c|c|c|c|c|c|}
\hline $\begin{array}{l}\text { Sample } \\
\text { ID }\end{array}$ & $\begin{array}{l}\text { Total tag } \\
\text { number }\end{array}$ & $\begin{array}{l}\text { Total tag } \\
\text { length, bp }\end{array}$ & $\begin{array}{l}\text { Mean length, } \\
\text { bp }\end{array}$ & $\begin{array}{l}\text {, Min length, } \\
\text { bp }\end{array}$ & $\begin{array}{l}\text { Max length, } \\
\text { bp }\end{array}$ \\
\hline EC-1d & 61639 & 25431211 & 413 & 261 & 514 \\
\hline EC-2d & 58044 & 24031647 & 414 & 328 & 514 \\
\hline$E C-3 d$ & 56668 & 23591214 & 416 & 319 & 483 \\
\hline EC-4d & 57618 & 23729682 & 412 & 254 & 463 \\
\hline EC-6d & 72425 & 29913072 & 413 & 232 & 501 \\
\hline ER-1f & 58732 & 24502833 & 417 & 317 & 483 \\
\hline ER-2f & 57826 & 24081958 & 416 & 297 & 492 \\
\hline ER-3f & 49790 & 20837327 & 419 & 327 & 465 \\
\hline ER-5f & 69731 & 29145798 & 418 & 335 & 522 \\
\hline ER-6f & 70596 & 29493081 & 418 & 321 & 445 \\
\hline SC-7 & 73715 & 30499096 & 414 & 219 & 509 \\
\hline SC-9 & 72662 & 30311299 & 417 & 245 & 458 \\
\hline SC-10 & 73502 & 30548412 & 416 & 216 & 436 \\
\hline SC-11 & 74671 & 31081964 & 416 & 234 & 444 \\
\hline SC-12 & 73643 & 30693287 & 417 & 269 & 434 \\
\hline SR-1 & 74642 & 31147919 & 417 & 258 & 520 \\
\hline SR-3 & 71455 & 29729693 & 416 & 220 & 463 \\
\hline SR-4 & 73513 & 30881879 & 420 & 214 & 473 \\
\hline SR-5 & 71684 & 29931980 & 418 & 270 & 432 \\
\hline SR-6 & 74163 & 31014432 & 418 & 231 & 478 \\
\hline
\end{tabular}

EC - Erhualian pig caecum, ER - Erhualian pig rectum, SC - Sushan pig caecum, SR - Sushan pig rectum

Table 3. Richness and diversity estimates of $16 \mathrm{~S}$ rRNA genes from the sequencing analysis in rectum and caecum of Erhualian and Sushan pigs

\begin{tabular}{|c|c|c|c|c|}
\hline \multirow{2}{*}{ Sample } & \multicolumn{2}{|c|}{ Species richness indices } & \multicolumn{2}{|c|}{ Species diversity indices } \\
\hline & Sobs & Chao1 & Shannon & Simpson \\
\hline$\overline{E C}$ & $649.4 \pm 107.39$ & $783.50 \pm 85.99$ & $4.39 \pm 0.47$ & $0.038 \pm 0.032$ \\
\hline ER & $699.8 \pm 32.39$ & $819.68 \pm 65.45$ & $4.62 \pm 0.15$ & $0.023 \pm 0.004$ \\
\hline SC & $755.4 \pm 62.28$ & $863.67 \pm 76.95$ & $4.94 \pm 0.11$ & $0.020 \pm 0.005$ \\
\hline SR & $840.4 \pm 52.33$ & $963.03 \pm 58.46$ & $4.51 \pm 0.17$ & $0.068 \pm 0.017$ \\
\hline
\end{tabular}

EC - Erhualian pig caecum, ER - Erhualian pig rectum, SC - Sushan pig caecum, SR - Sushan pig rectum

Species composition analysis. Figure 1 reveals that 1397 different OTUs were distributed in rectum and caecum of Erhualian and Sushan pigs. In total 842 OTUs were shared by all of the samples, and the number of unique OTUs in rectum of Sushan pigs was the highest. The microbial populations of Lactobacillus $(9.61 \%)$ were the largest in rectum

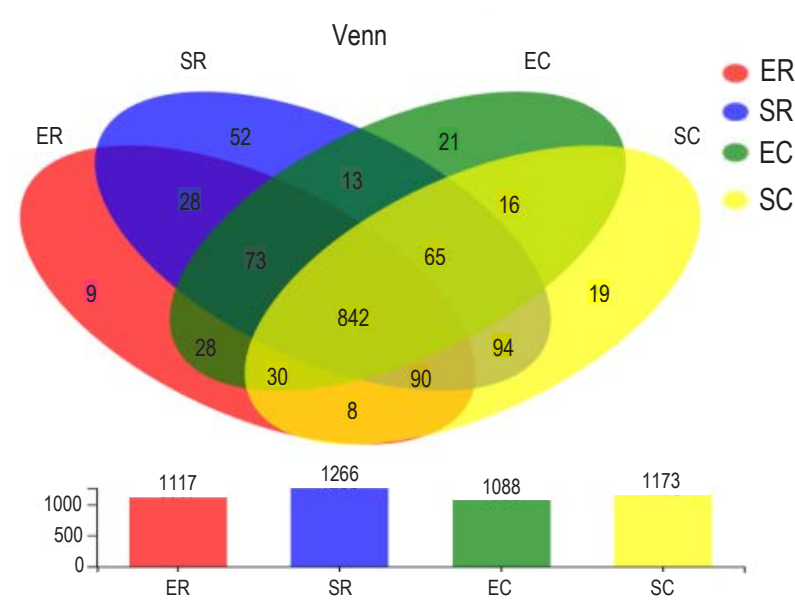

Figure 1. Venn diagrams of the operational taxonomic units (OUT) EC - Erhualian pig caecum, ER - Erhualian pig rectum, SC - Sushan pig caecum, SR - Sushan pig rectum

of Erhualian pigs (Figure 2A), Ruminococcaceae UCG-005 (10.89\%) in caecum of Erhualian pigs (Figure 2B), Streptococcus (24.89\%) in rectum of Sushan pigs (Figure 2C) and Lactobacillus (6.83\%) in caecum of Sushan pigs (Figure 2D), respectively. Among the dominant bacteria, Lachnospiraceae XPB1014 was the unique species identified in rectum of Erhualian pigs; Coprococcus, Phascolarctobacterium and Lachnospiraceae NK4A136 in caecum of Erhualian pigs; Fusobacterium in caecum of Sushan pigs; and Prevotella and Ruminococcaceae NK4A214 in rectum of Sushan pigs, respectively. Alloprevotella, Romboutsia, Christensenellaceae R-7 and Rikenellaceae RC9 were among the dominant bacteria in caecum of Erhualian and Sushan pigs. Prevotellaceae UCG-003 was not found in rectum of Erhualian pigs, while Prevotellaceae NK3B31 was not found in caecum of this breed. Clostridium was not in the dominant bacteria found in caecum of Sushan pigs.

Species difference analysis. The significance of microbial compositional differences in caecum and rectum of Erhualian and Sushan pigs was tested (Figure 3). The results showed that four genera were significantly different in caecum of Erhualian and Sushan pigs, and Ruminococcaceae UCG-005 and Christensenellaceae R-7 exhibited highly significant differences (Figure 3A); three genera were significantly different in rectum of Erhualian and Sushan pigs, and Streptococcus exhibited highly significant differences (Figure 3B); seven genera were significantly different in caecum and rectum of Erhualian pigs, and Christensenellaceae R-7 exhibited highly significant differences (Figure 3C); four genera were significantly different in caecum and rectum of Sushan pigs, and Streptococcus and 
A Community analysis pieplot on genus level: ER

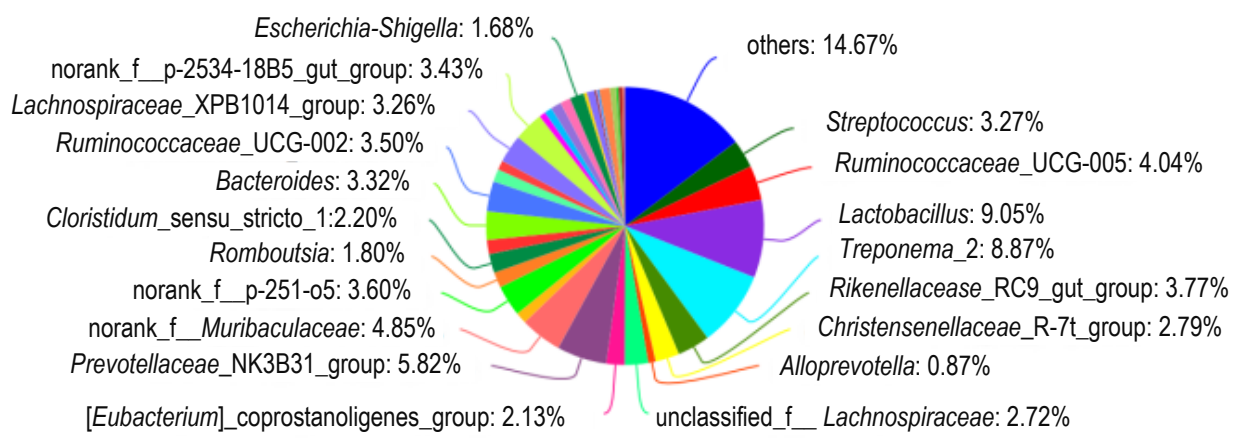

B Community analysis pieplot on genus level: EC

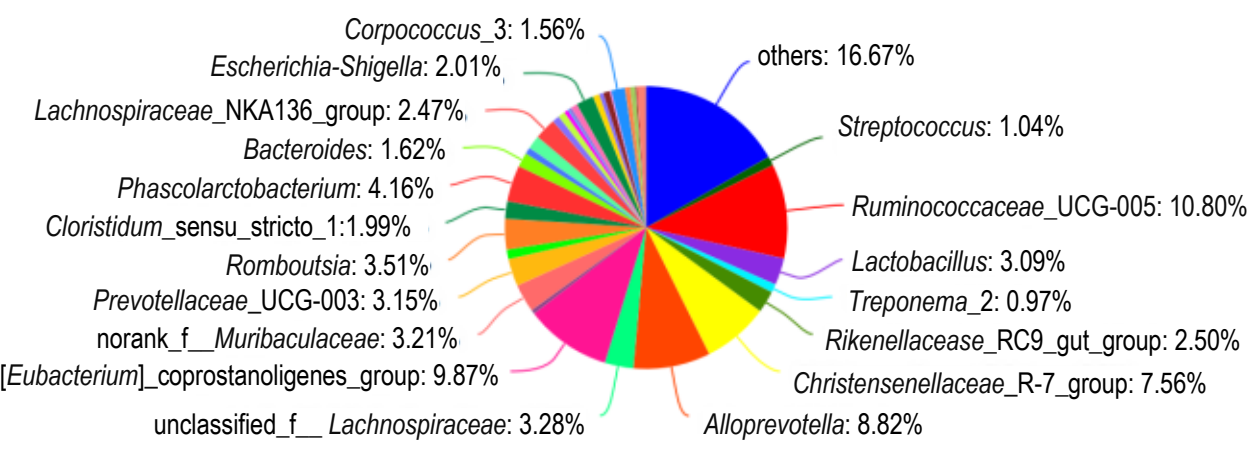

C Community analysis pieplot on genus level: SR

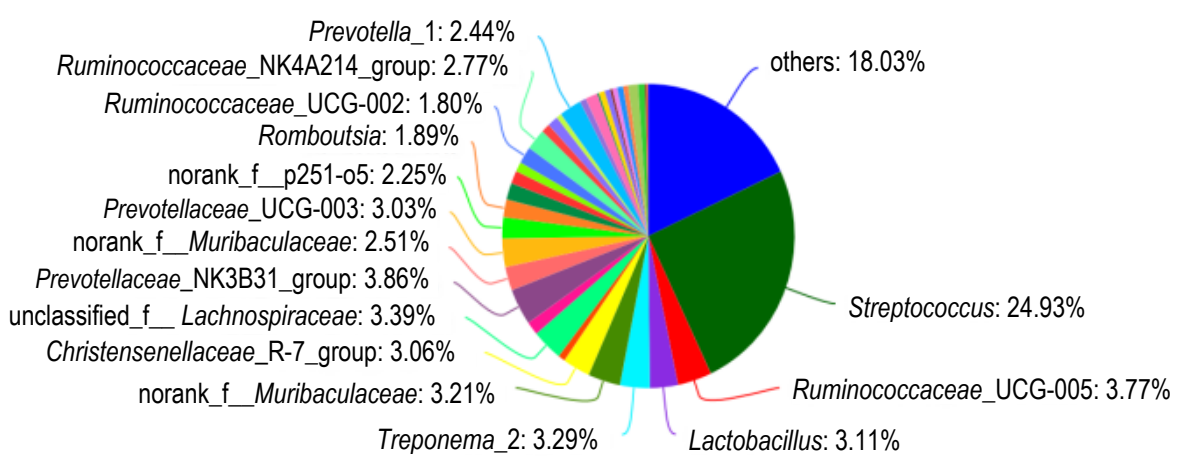

D Community analysis pieplot on genus level: SC

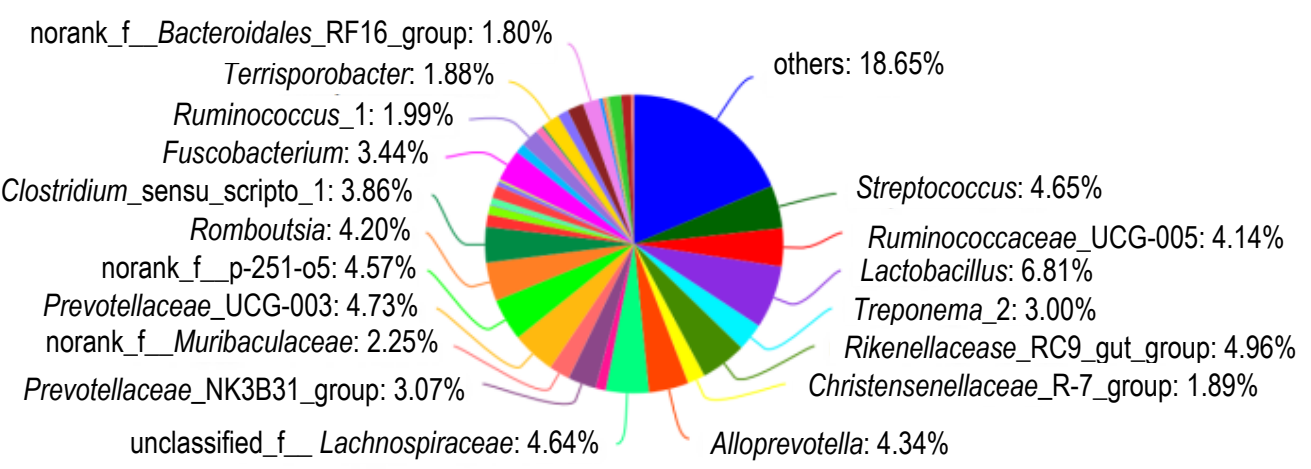

Figure 2. The community pieplot on genus level in rectum and caecum of Erhualian and Sushan pigs EC - Erhualian pig caecum, ER - Erhualian pig rectum, SC - Sushan pig caecum, SR - Sushan pig rectum 
A

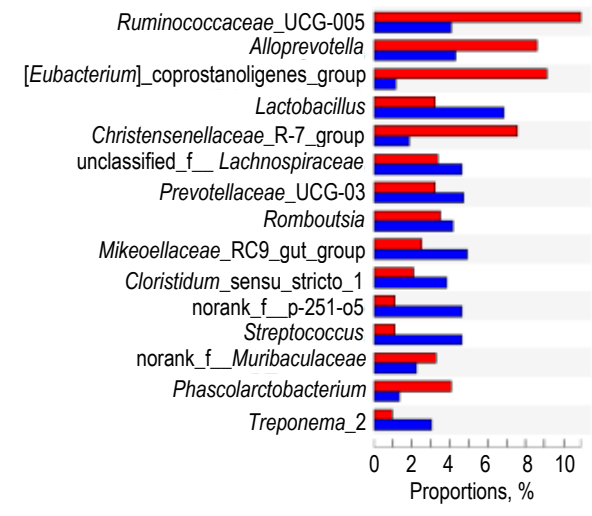

B

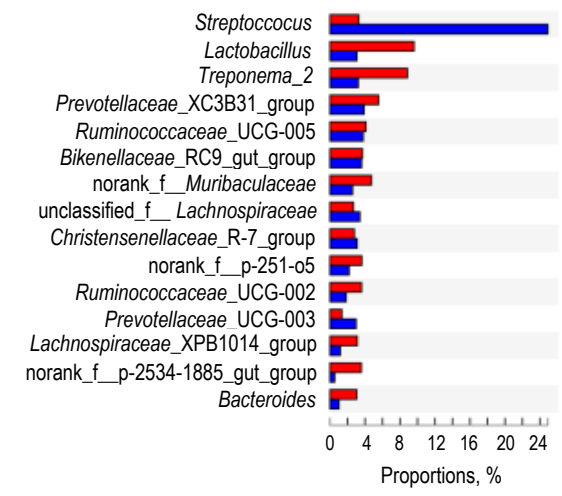

C

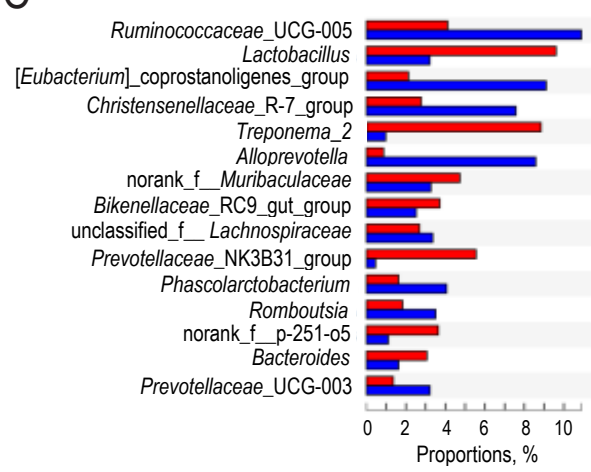

D

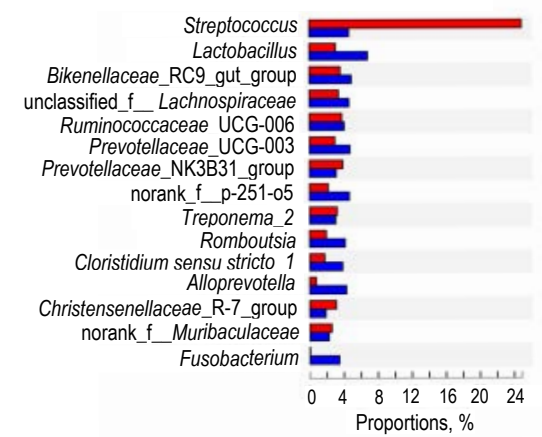

95 confidence intervals

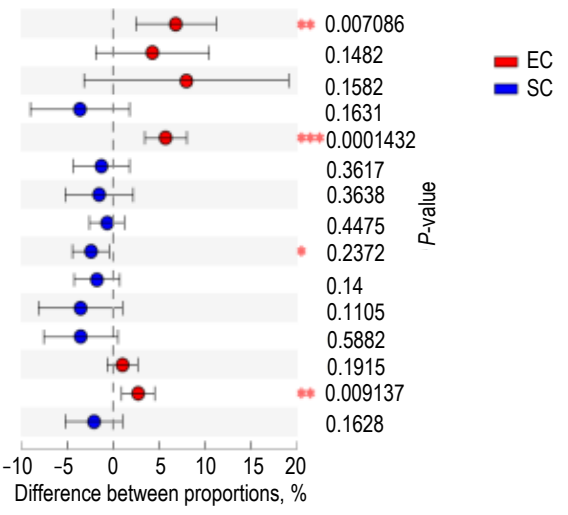

95 confidence intervals

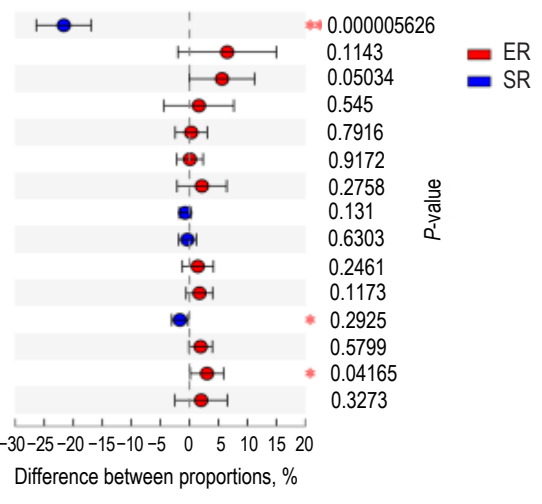

95 confidence intervals

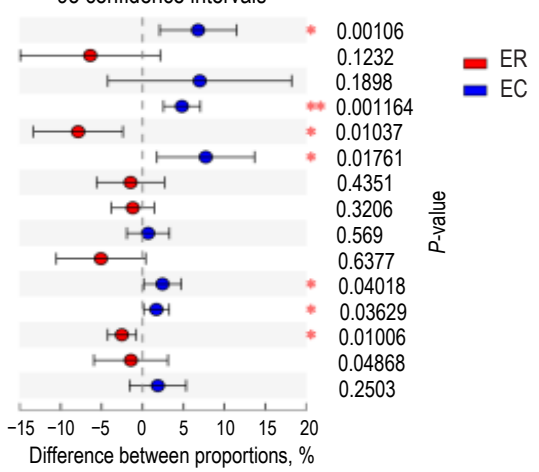

95 confidence intervals

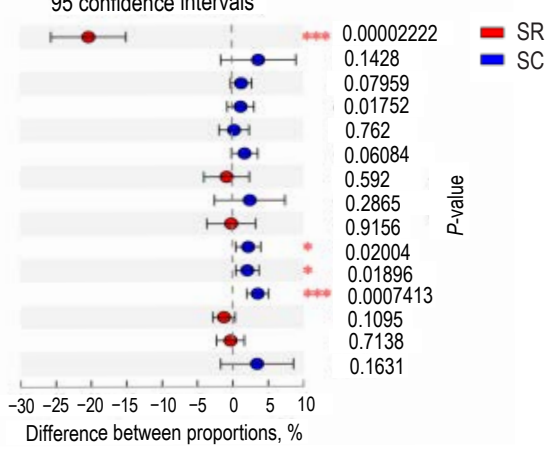

Figure 3. Phylotypes significantly different between EC and SC (A), ER and SR (B), ER and EC (C), SR and SC (D) groups at the genus level EC - Erhualian pig caecum, ER - Erhualian pig rectum, SC - Sushan pig caecum, SR - Sushan pig rectum; statistical analysis was performed by the Student's t-test; $\mathrm{n}=5$, in each group; ${ }^{*} P \leq 0.05,{ }^{* *} P \leq 0.01$ and ${ }^{* * *} P \leq 0.001$ 
A

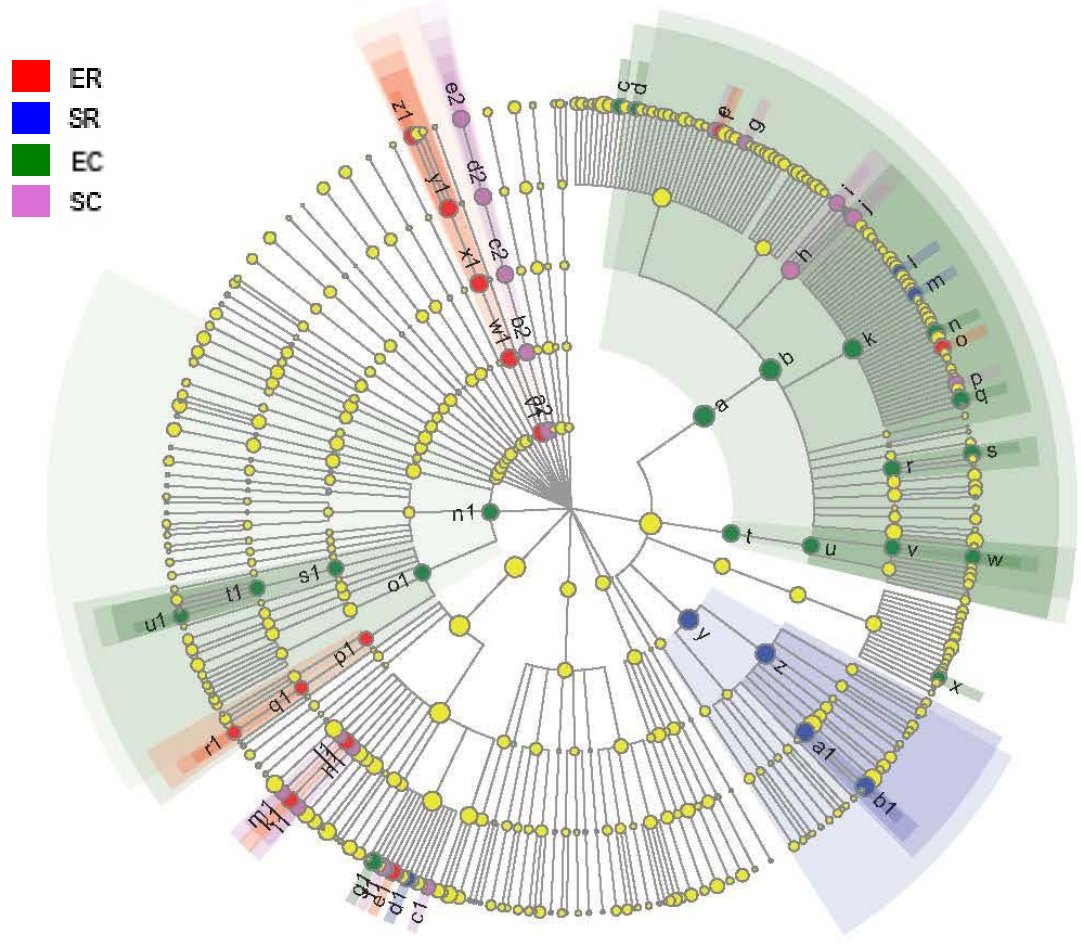

B

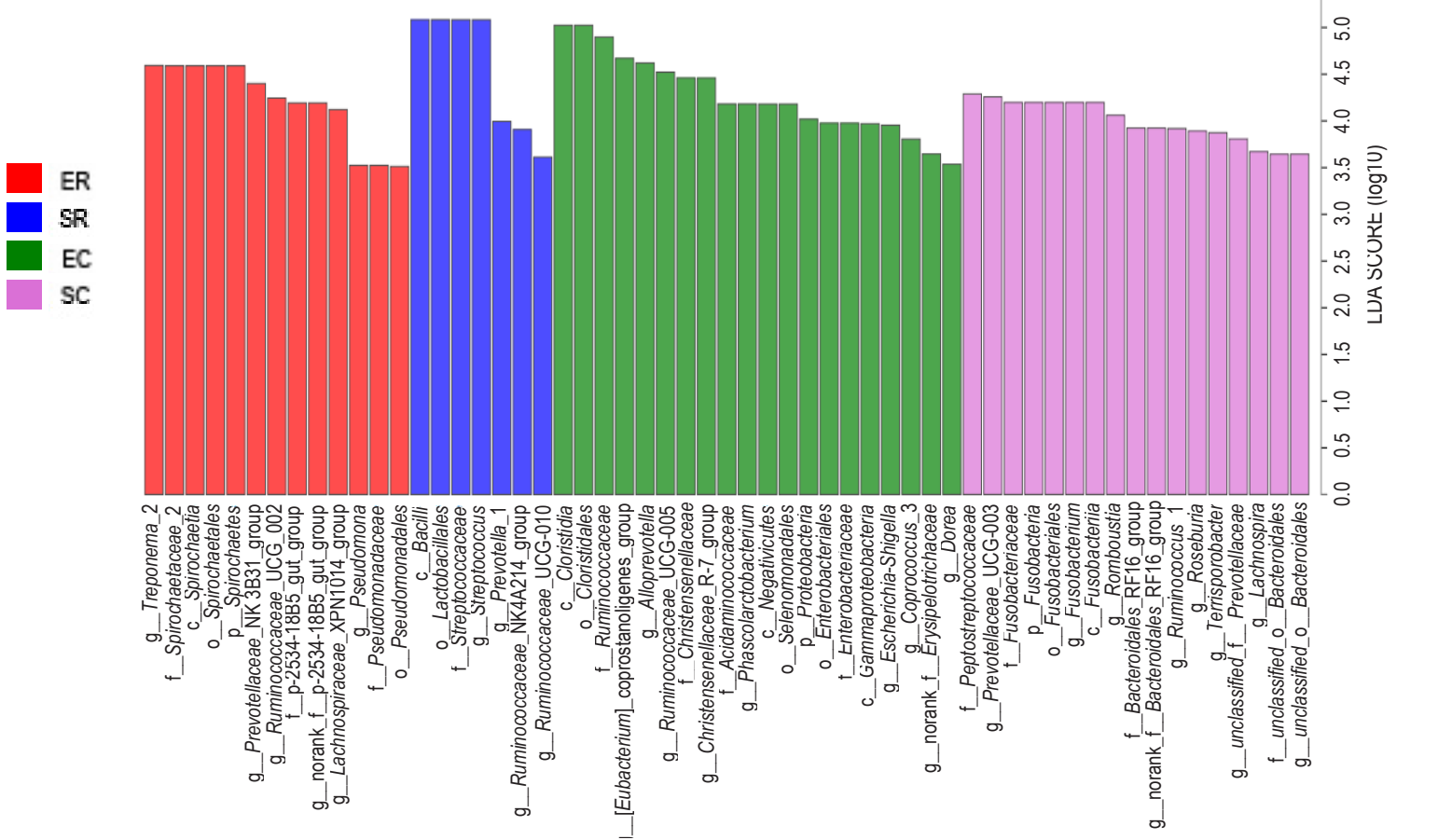

Figure 4. Cladogram (A) and LDA (B) analyses of bacterial communities associated with different portions of intestines in Sushan and Erhualian pigs. Different-coloured regions represent different intestinal parts of two breeds (red, ER; blue, SR; green, EC; pink, SC). Circles indicate phylogenetic levels from phylum to genus. The diameter of each circle is proportional to the abundance of the group. EC - Erhualian pig caecum, ER - Erhualian pig rectum, SC - Sushan pig caecum, SR - Sushan pig rectum

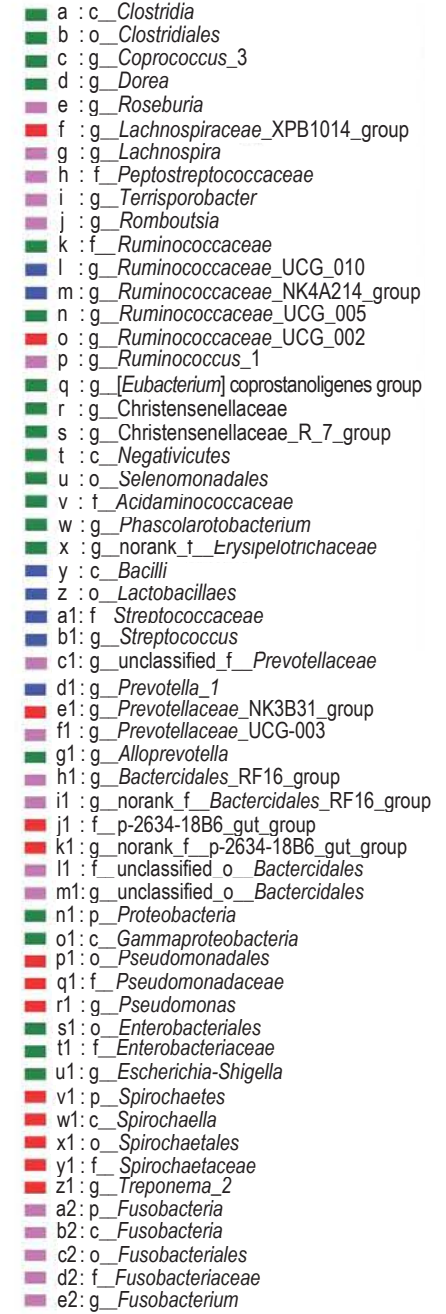

- b : o_Clostridiales

- d : g_Dorea

을

i : $\mathrm{g}$ Terrisporobacter

=

: g_Ruminococcaceae UCG 010

n:

- $0:$ g_Ruminococcacea

- q : g_[Eubacterium] coprostanoligenes group

: g_Christensenellaceae

: C_Negativicutes

$x: g$ norank ${ }^{\dagger}$ tryspelotrichaceae

$z: 0$ Lactobacillaes

b1: Streptococcaceae

c1: g_unclassified f

e1:g Prevotellaceae NK3B31 group

g1: g Alloprevotella

i1: g_Bactercidales_RF16_group 216 group

- 11 : f unclassified o Bactercidales

n1:p_Proteobacteria

p1:- _ _ Pammaproteobacterianonadales

- $\mathrm{q} 1 \mathrm{f}$ _-Pseudomonadaceae

r1:g_Pseudomonas

11. Enterobacteriaceae

$v 1: p \_$Spirochaetes

x1:0_Spirochaetales

$\mathrm{z} 1$ : g_ Treponema_2$$
\text { C; pink. }
$$ 
Alloprevotella exhibited highly significant differences (Figure 3D). Cladogram exhibited a distinct phylogenetic distribution of the bacterial lineages in different intestinal segments of Erhualian and Sushan pigs (Figure 4A). Indicator bacteria with LDA scores of 3.5 in bacterial communities were associated with different intestinal parts of two breeds (Figure 4B). In rectum of Erhualian pigs, enriched bacterial groups included Treponema (genus), Spirochaetes (from phylum to family), Prevotellaceae (genus), Ruminococcaceae (genus), Lachnospiraceae and Pseudomonadales (from order to genus). In rectum of Sushan pigs, enriched bacterial groups included Bacilli (class), Lactobacillales (order) and Streptococcaceae (from family to genus). In caecum of Erhualian pigs, Clostridia (from class to order), Ruminococcaceae (from family to genus), Alloprevotella (genus), Christensenellaceae (from family to genus) and Enterobacteriales (from order to genus) were significantly enriched. In caecum of Sushan pigs, enriched bacterial groups included Fusobacteria (from phylum to genus), Bacteroidales (from family to genus), Peptostreptococcaceae (family) and Prevotellaceae UCG-003.

Sample comparison analysis. The bacterial community structures of different intestinal segments of the two breeds of pigs were clearly separated, and two coordinates (PC1 and PC2) explained $41.37 \%$ of the total variation of bacteria (Figure $5 \mathrm{~A}$ ). In addition, the Jensen-Shannon distance was calculated according to the abundance of the microflora at the genus level and clustered by PAM (partitioning around medoids) to obtain the optimal clustering $\mathrm{K}$ value of 3 . Then, the PCA results were visually displayed. The analysis of microflora typing showed three types of intestinal microflora clustering (Figure 5B). Intestinal type 1 was the Streptococcus intestinal type, which was mainly present in rectum of Sushan pigs; intestinal type 2 was the Lactobacillus intestinal type, which was mainly present in rectum of Erhualian pigs and caecum of Sushan pigs; and intestinal type 3 was the Ruminococcaceae UCG-005 intestinal type, which was mainly present in caecum of Erhualian pigs.

Community function prediction and pathway enrichment analyses. Using the present OTU data, PICRUS was applied to determine the potential pathway enrichment of intestinal samples via annotation against the KEGG database. In all samples, the majority of OTUs were assigned to 24 gene families, which were mainly involved in carbohydrate transport and metabolism, general function prediction, amino acid transport and metabolism, transcription, replication, recombination and repair,
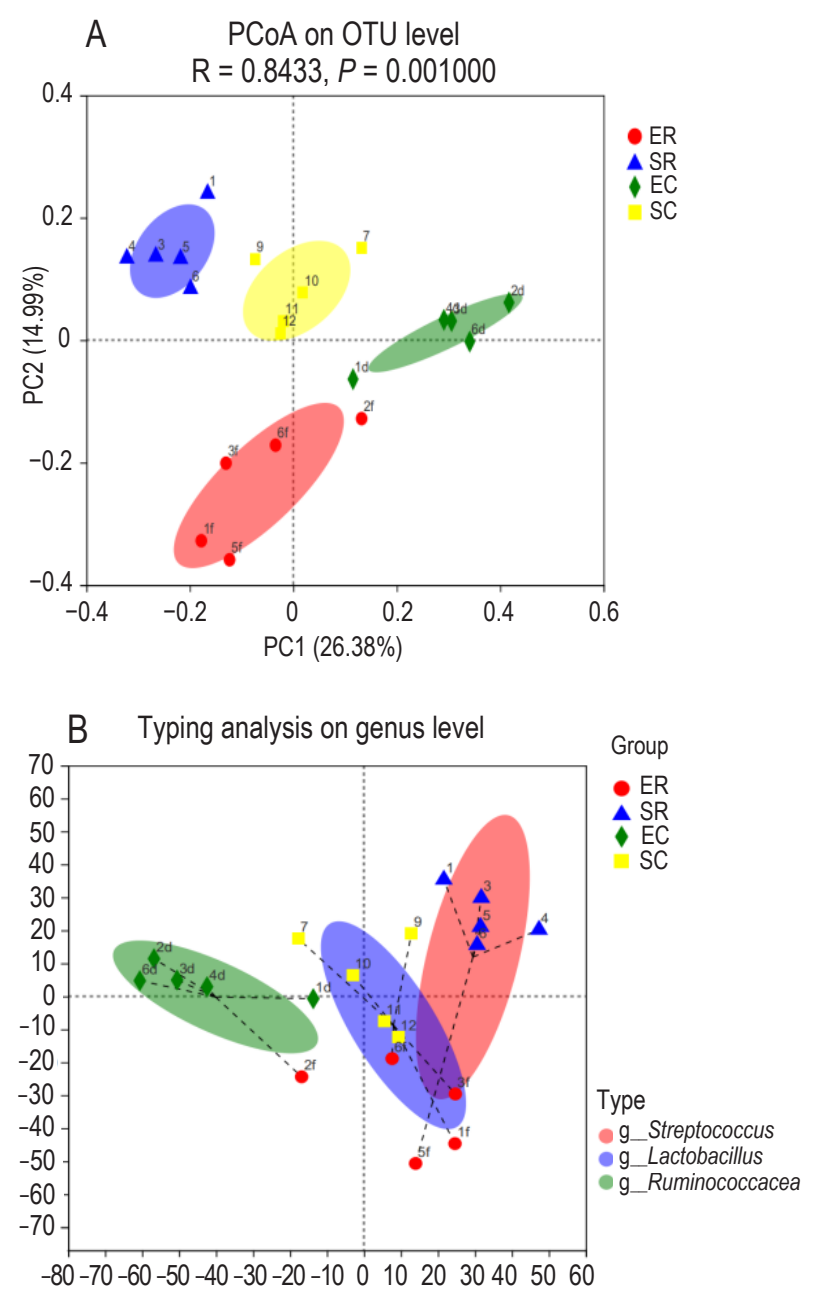

Figure 5. Principal coordinate analysis (PCA) plots $(A)$ and microflora typing $(B)$ of bacterial communities in rectum and caecum of Erhualian and Sushan pigs

EC - Erhualian pig caecum, ER - Erhualian pig rectum, SC - Sushan pig caecum, SR - Sushan pig rectum

translation, ribosomal structure and biogenesis. Compared with breed composition, the COG functional composition of all the samples was relatively similar. No significant difference was found in different samples (Figure 6). The prevalence of pathways at the KEGG 1 class level was similar among different samples, and their abundance values of metabolism were the highest (Figure 7A). The results of pathway level 2 revealed that relatively few microbial communities in rectum of Erhualian and Sushan pigs were involved in amino acid metabolism, biosynthesis of other secondary metabolites, cell motility, cellular processes and signalling, energy metabolism, environmental adaptation, enzyme families, folding, sorting and degradation, genetic information processing, immune system, lipid metabolism, metabolism of cofactors and vitamins, metabolism of other amino acids, nervous system and nucleotide metabolism. However, in rectum of Erhualian and Sushan pigs, the abundance values 

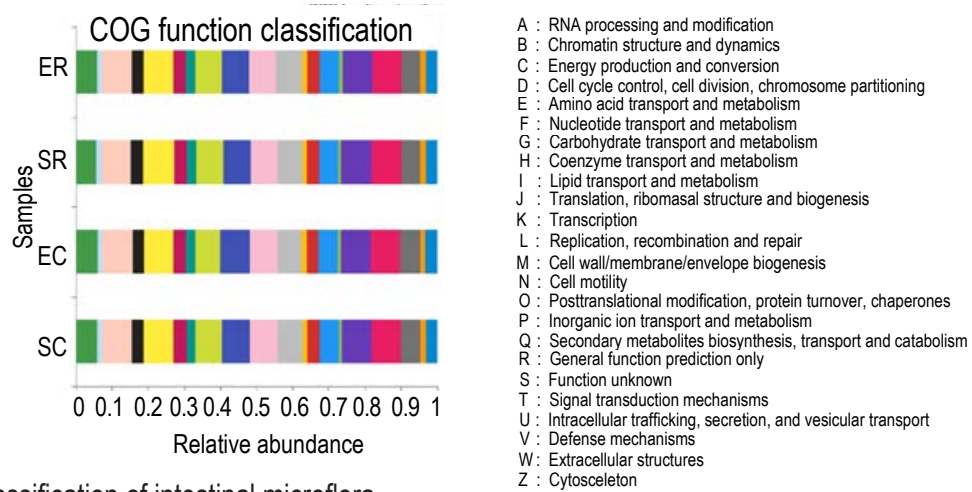

Figure 6. COG functional classification of intestinal microflora 
of the digestive system were significantly higher than those in caecum of Erhualian and Sushan pigs. On the other hand, in rectum and caecum of Erhualian pigs relatively more microbial communities were involved in cell growth, cell death and the endocrine system than those in Sushan pigs (Figure 7B).

\section{Discussion}

The main function of intestinal microorganisms is to help animals digest and utilize nutrients in the diet, assist host metabolism, make nutrients better used by animals, provide nutrition for intestinal epithelial cells, strengthen their immune and disease resistance functions, and help the host resist the invasion of harmful pathogens (Kim et al., 2011; Thaiss et al., 2016). The main factors affecting the changes of intestinal microflora are the host itself, dietary factors and the interaction of microflora. Among them, the host itself is the most direct and important factor.

There are great differences in the structure of intestinal microflora and the abundance of microflora among different breeds of pigs. Some studies have shown that the intestinal microflora of pigs is affected by the genetic background, and there are differences in the intestinal microflora of different breeds of pigs. Yang et al. (2014) found that the number of total bacteria, Firmicutes and Bacteroidetes in the faeces of Chinese local pig breeds (Bama mini, Meishan and Erhualian pigs), was significantly higher than that of Duroc pigs. Pig breeds had a significant effect on the structure of intestinal microflora of adult sows. Bama mini pigs had the most abundant intestinal microflora. There was a significant difference between Chinese local pig breeds and Duroc pig breeds. Xiao et al. (2018) found that the microbial diversity in caecum and colon was higher than that in duodenum, jejunum and ileum in Jinhua and Landrace pigs. In this study, the diversity of intestinal microflora in two pig breeds was similar, but the abundance of specific intestinal microflora was different. The relative abundance of rectal microflora was higher than that of caecum, and there were differences between the two breeds. The abundance of rectal and caecum microflora in Sushan pigs was higher than that of Erhualian pigs, but the rectal microflora polymorphism in Sushan pigs was lower than that in Erhualian pigs. The caecum microflora diversity in Sushan pigs was higher than that in Erhualian pigs. The above results indicated that the abundance and diversity of microorganisms in the pig intestine were highly correlated with breeds and specific intestinal segments.

At the phylum level, it was found that the dominant bacteria in caecum and rectum of Erhualian and Sushan pigs were Firmicutes, Acidobacteria and Bacteroides, but the expression abundance of different bacteria was different. Firmicutes and Bacteroidetes in Erhualian pigs were higher than those in Sushan pigs. Some studies have found that the abundance of Firmicutes in Jinhua pigs was higher than that in Duroc, Yorkshire and Landrace pigs, but the abundance of Bacteroidetes was lower (Pajarilla et al., 2014; Yang et al., 2018). Bacteroides, Firmicutes, Spirochaetae and Proteus were the dominant flora in the faeces of Tibetan, Rongchang and Yorkshire pigs. The abundance of Firmicutes in Tibetan and Rongchang pigs was higher than that in Yorkshire pigs, but the abundance of Bacteroides was lower (Diao et al., 2016). Firmicutes and Bacteroidetes are associated with crude fibre digestion and carbohydrate degradation. The ratio of Firmicutes/ Bacteroidetes can reflect the host's lipid metabolism, and a higher ratio will cause obesity and other complications (Kim and Isaacson, 2015; Mathur and Barlow, 2015). This may be because Erhualian, Tibetan and Rongchang pigs are obese ones that have a strong ability to deposit fat and digest crude fibre.

At the genus level, it was found that the abundance of Christensenellaceae, Ruminococcaceae, Alloprevotella, Phascolarctobacterium, Treponema, Bacteroides in Erhualian pigs was significantly higher than that in Sushan pigs, and the abundance of Rikenellaceae, Streptococcus, Prevotellaceaein in Sushan pigs was significantly higher than that in Erhualian pigs. The results of this study are different from previous ones. Guo et al. (2008) found that the abundance of Bacteroides in the obese pig is less than that in lean pig, and the increase of Bacteroides has a negative impact on body weight (Simpson et al., 1999; Dowarah et al., 2017). This may be due to the fact that Sushan pig is a new hybrid breed of Erhualian and Yorkshire pigs, and belongs to the obese pig. The abundance of Streptococcus in rectum of Sushan pig was significantly higher than that in Erhualian pig. In this context, our results are not different to previous ones. Xiao et al. (2018) also found that the abundance of Streptococcus in jejunum, ileum and colon of Jinhua pigs was higher than that in Landrace pigs, indicating that Streptococcus has various specificity. Streptococcus is related to inflammation and diseases. Whether disease resistance of different pig breeds is related to the abundance of Streptococcus in the intestine remains to be studied. 
Christensenellaceae, Ruminocaceae, Alloprevotella and Phascolarctobacterium are all closely related to fat deposition (Bian et al., 2016; Yang et al., 2018). Erhualian pig is a typical Chinese local obese pig with high-fat deposition ability. Whether these bacteria affect fat deposition in Erhualian pigs needs further study. The results of this study further confirmed the existence of specific microbial community diversity and abundance in different breeds of pigs. The microbial community diversity and abundance in the pig intestine were closely related to fat deposition and nutrient absorption of pigs.

\section{Conclusions}

In summary, comparisons between different intestinal segments of the two pig breeds showed distinct structural compositions and predicted functions of microbial communities. Christensenellaceae R-7, Ruminococcaceae UCG-005, Alloprevotella and Phascolarctobacterium were dominant in caecum of Erhualian pigs, which were associated with fat deposition and crude fibre digestion in pigs. These results may indicate that Erhualian pigs might have stronger fat deposition and crude fibre tolerance than Sushan pigs. Streptococcus number in the intestine of Sushan pigs was significantly higher than that in Erhualian pigs. Whether the stronger disease resistance of Sushan pig is related to the abundance of Streptococcus in the intestine needs further study.

\section{Funding}

This study was supported by China Agriculture Research System of MOF and MARA.

\section{Conflict of interest}

The authors declare that there is no conflict of interest.

\section{References}

Bergen W.G., 2015. Small-intestinal or colonic microbiota as a potential amino acid source in animals. Amino Acids 47, 251-258, https://doi.org/10.1007/s00726-014-1875-z

Bian G., Ma S., Zhu Z. et al., 2016. Age, introduction of solid feed and weaning are more important determinants of gut bacterial succession in piglets than breed and nursing mother as revealed by a reciprocal cross-fostering model. Environ. Microbiol. 18, 1566-1577, https://doi.org/10.1111/14622920.13272

Chen S., Zhou Y., Chen Y., Gu J., 2018. fastp: an ultra-fast all-in-one FASTQ preprocessor. Bioinformatics 34, i884-i890, https:// doi.org/10.1093/bioinformatics/bty560
Choy Y.Y., Quifer-Rada P., Holstege D.M., Frese S.A., Calvert C.C Mills D.A., La-muela-Raventos R.M., Waterhouse A.L., 2014. Phenolic metabolites and substantial microbiome changes in pig feces by ingesting grape seed proanthocyanidins. Food Funct. 5, 2298-2308, https://doi.org/10.1039/c4fo00325j

Diao H., Yan H.L., Xiao Y. et al., 2016. Intestinal microbiota could transfer host Gut characteristics from pigs to mice. BMC Microbiol. 16, 238, https://doi.org/10.1186/s12866-0160851-z

Dowarah R., Verma A.K., Agarwal N., Patel B.H.M., Singh P., 2017. Effect of swine based probiotic on performance, diarrhoea scores, intestinal microbiota and gut health of growerfinisher crossbred pigs. Livest. Sci. 195, 74-79, https://doi. org/10.1016/j.livsci.2016.11.006

Du J., Yuan Z., Ma Z., Song J., Xie X., Chen Y., 2014. KEGG-PATH: Kyoto encyclopedia of genes and genomes-based pathway analysis using a path analysis model. Mol. BioSyst. 10, 2441-2447, https://doi.org/10.1039/c4mb00287c

Edgar R.C., 2013. UPARSE: Highly accurate OTU sequences from microbial amplicon reads. Nat. Methods 10, 996-998, https:// doi.org/10.1038/nmeth.2604

Guo X., Xia X., Tang R., Zhou J., Zhao H., Wang K., 2008. Development of a real-time PCR method for Firmicutes and Bacteroidetes in faeces and its application to quantify intestinal population of obese and lean pigs. Lett. Appl. Microbiol. 47, 367-373, https://doi.org/10.1111/j.1472-765X.2008.02408.x

Kemp P.F., Aller J.Y., 2004. Bacterial diversity in aquatic and other environments: what $16 \mathrm{~S}$ rDNA libraries can tell us. FEMS Microbiol. Ecol. 47, 161-177, https://doi.org/10.1016/S01686496(03)00257-5

Kim H.B., Borewicz K., White B.A., Singer R.S., Sreevatsan S., Tu Z.J., Isaacson R.E., 2011. Longitudinal investigation of the age-related bacterial diversity in the feces of commercial pigs. Vet. Microbiol. 153, 124-133, https://doi.org/10.1016/j. vetmic.2011.05.021

Kim H.B., Isaacson R.E., 2015. The pig gut microbial diversity: understanding the pig gut microbial ecology through the next generation high throughput sequencing. Vet. Microbiol. 177, 242-251, https://doi.org/10.1016/j.vetmic.2015.03.014

Langille M.G., Zaneveld J., Caporaso J.G. et al., 2013. Predictive functional profiling of microbial communities using 16S rRNA marker gene sequences. Nat. Biotechnol. 31, 814-821, https://doi.org/10.1038/nbt.2676

Magoč T., Salzberg S.L., 2011. FLASH: Fast length adjustment of short reads to improve genome assemblies. Bioinformatics 27, 2957-2963, https://doi.org/10.1093/bioinformatics/btr507

Mathur R., Barlow G.M., 2015. Obesity and the microbiome. Expert Rev. Gastroenterol. Hepatol. 9, 1087-1099, https://doi.org/10 .1586/17474124.2015.1051029

Niu Q., Li P., Hao S., Kim S.W., Du T., Hua J., Huang R., 2019. Characteristics of gut microbiota in sows and their relationship with apparent nutrient digestibility. Int. J. Mol. Sci. 20, 870, https://doi.org/10.3390/ijms20040870

Pajarillo E.A.B., Chae J.P., Balolong M.P., Kim H.B., Seo K.-S., Kang D.-K., 2014. Pyrosequencing-based analysis of fecal microbial communities in three purebred pig lines. J. Microbiol. 52, 646-651, https://doi.org/10.1007/s12275014-4270-2

R Core Team, 2020. R: a Language and Environment for Statistical Computing. R Foundation for Statistical Computing. Vienna (Austria), https://www.r-project.org

Robinson M.D., McCarthy D.J., Smyth G.K., 2010. edgeR: a Bioconductor package for differential expression analysis of digital gene expression data. Bioinformatics 26, 139-140, https://doi.org/10.1093/bioinformatics/btp616 
Segata N., Izard J., Waldron L., Gevers D., Miropolsky L., Garrett W.S., Huttenhower C., 2011. Metagenomic biomarker discovery and explanation. Genome Biol. 12, R60, https://doi.org/10.1186/ gb-2011-12-6-r60

Schloss P.D., Westcott S.L., Ryabin T. et al., 2009. Introducing mothur: open-source, platform-independent, communitysupported software for describing and comparing microbial communities. Appl. Environ. Microbiol. 75, 7537-7541, https://doi.org/10.1128/AEM.01541-09

Simpson J.M., McCracken V.J., White B.A., Gaskins H.R., Mackie R.I., 1999. Application of denaturant gradient gel electrophoresis for the analysis of the porcine gastrointestinal microbiota. J. Microbiol. Methods 36, 167-179, https://doi.org/10.1016/ S0167-7012(99)00029-9

Thaiss C.A., Zmora N., Levy M., Elinav E., 2016. The microbiome and innate immunity. Nature 535, 65-74, https://doi.org/10.1038/ nature 18847

White J.R., Nagarajan N., Pop M., 2009. Statistical methods for detecting differentially abundant features in clinical metagenomic samples. PLoS Comp. Biol. 5, e1000352, https://doi.org/10.1371/journal.pcbi.1000352
Woting A., Pfeiffer N., Loh G., Klaus S., Blaut M., 2014. Clostridium ramosum promotes high-fat diet-induced obesity in gnotobiotic mouse models. mBio 5, e01530-14, https://doi. org/10.1128/mBio.01530-14

Xiao Y., Kong F., Xiang Y., Zhou W., Wang J., Yang H., Zhang G., Zhao J., 2018. Comparative biogeography of the gut microbiome between Jinhua and Landrace pigs. Sci. Rep. 8, 5985, https://doi.org/10.1038/s41598-018-24289-z

Yang H., Xiao Y., Wang J., Xiang Y., Gong Y., Wen X., Li D., 2018. Core gut microbiota in Jinhua pigs and its correlation with strain, farm and weaning age. J. Microbiol. 56, 346-355, https://doi.org/10.1007/s12275-018-7486-8

Yang L., Bian G., Su Y., Zhu W., 2014. Comparison of faecal microbial community of Lantang, Bama, Erhualian, Meishan, Xiaomeishan, Duroc, Landrace, and Yorkshire sows. AsianAustralas. J. Anim. Sci. 27, 898-906, https://doi.org/10.5713/ ajas.2013.13621 